An improved analysis of the SSHI interface in piezoelectric energy harvesting

This article has been downloaded from IOPscience. Please scroll down to see the full text article.

2007 Smart Mater. Struct. 162253

(http://iopscience.iop.org/0964-1726/16/6/028)

The Table of Contents and more related content is available

Download details:

IP Address: 140.112.113.225

The article was downloaded on 22/12/2008 at 01:30

Please note that terms and conditions apply. 


\title{
An improved analysis of the SSHI interface in piezoelectric energy harvesting
}

\author{
Y C Shu ${ }^{1,3}$, I C Lien ${ }^{1}$ and W J Wu ${ }^{2}$ \\ ${ }^{1}$ Institute of Applied Mechanics, National Taiwan University, Taipei 106, Taiwan, \\ Republic of China \\ ${ }^{2}$ Department of Engineering Science and Ocean Engineering, National Taiwan University, \\ Taipei 106, Taiwan, Republic of China \\ E-mail: yichung@spring.iam.ntu.edu.tw
}

Received 29 April 2007, in final form 15 August 2007

Published 9 October 2007

Online at stacks.iop.org/SMS/16/2253

\begin{abstract}
This paper provides an analysis for the performance evaluation of a piezoelectric energy harvesting system using the synchronized switch harvesting on inductor (SSHI) electronic interface. In contrast with estimates based on a variety of approximations in the literature, an analytic expression of harvested power is derived explicitly and validated numerically for the SSHI system. It is shown that the electrical response using an ideal SSHI interface is similar to that using the standard interface in a strongly coupled electromechanical system operated at short circuit resonance. On the other hand, if the SSHI circuit is not ideal, the performance degradation is evaluated and classified according to the relative strength of coupling. It is found that the best use of the SSHI harvesting circuit is for systems in the mid-range of electromechanical coupling. The degradation in harvested power due to the non-perfect voltage inversion is not pronounced in this case, and a new finding shows that the reduction in power is much less sensitive to frequency deviations than that using the standard technique.
\end{abstract}

(Some figures in this article are in colour only in the electronic version)

\section{Introduction}

The need to power remote systems or embedded devices independently, coupled with advancements in low-power electronics, has motivated many research efforts to focus on producing electrical energy from various ambient energy sources. These include solar power, thermal gradients and vibration. Among these energy scavenging sources, parasitic mechanical vibration is a potential power source that is abundant enough to be of use, is easily accessible through microelectromechanical systems (MEMS) manufacturing processes for conversion to electrical energy, and is ubiquitous in applications from small household appliances to large infrastructure elements [50, 52].

While there are several options for transmitting vibration energy into electrical energy, vibration-based piezoelectric converters have received much attention as transducers $[18,31]$, since they have high electromechanical coupling,

3 Author to whom any correspondence should be addressed. require no external voltage source, and are particularly attractive for use in MEMS [10, 17, 22, 25, 38]. As a result, piezoelectric elements for scavenging energy from ambient vibration sources have been used in various types of structures to serve specific purposes, including the use of resonant piezoelectric-based structures of cantilever beam configuration $[1,11,14,23,40,51,54]$ as well as plate (membrane) configuration $[7,8,16,29,30,66]$. Other harvesting schemes include the use of long strips of piezoelectric polymers in ocean or river-water flows $[2,61]$, the use of piezoelectric 'cymbal' transducers [27, 28], and the use of piezoelectric windmills for generating electrical power from wind energy [45, 46]. The application to structural health monitoring with vibration powered piezoelectric sensors can be found in $[15,37,41]$.

Fundamental research on the study of optimal AC power output has been reported extensively in the recent literature $[12,13,26,44,60,65]$. In most of the work reported above, the vibration source is typically represented by a single harmonic signal, and an energy scavenger is required 
to resonate mechanically at a frequency tuned to that of the driving source to which it is attached, in order to generate the maximum electrical energy. As a frequency mismatch of only a few percent may result in a significant drop in power output, methods to adjust the resonance frequency to match the driving vibrations across a range of frequencies have been proposed by $[6,34,53]$. Fundamental investigations concerning the conversion efficiency by piezoelectric power generators include the early works by $[19,62,63]$ and the recent works by $[9,48,49,56]$. Another fundamental study relating energy harvesting to electrically induced damping can be found in [36]. It is shown that the damping added to a vibrating structure is attributed to the removal of electrical energy from the system, and an explicit expression of this induced damping is provided in the case of a weakly coupled electromechanical system. In addition, the power harvesting system is demonstrated to work similarly to a shunt damping system [35] except that the energy is stored instead of dissipated.

The research works cited above focus mainly on developing optimal energy harvesting structures so only AC power output is considered there. However, the electrical outputs of these devices in many cases are too small to power electrical devices directly. Thus, the methods of accumulating and storing parasitic energy for intermittent use are also the key to developing self-powered systems $[58,59]$. In addition, a vibrating piezoelectric element generates an $\mathrm{AC}$ voltage, while the electronic device in many practical applications requires a stabilized DC voltage. As a result, the electrical interface connecting the piezoelectric element and the terminal electrical load is needed to ensure electrical compatibility. Power optimization schemes therefore depend not only on the mechanical solicitation, but also on the specific type of electronic interface circuit chosen in the energy harvesting system. However, much work has addressed optimal AC power flow, while little has considered AC/DC power output until recently. Ottman et al [42, 43] have used the linear load impedance adaptation to develop highly efficient electrical circuits to store the generated charge. They have claimed that at high levels of excitation the power output can be increased by as much as $400 \%$. But the linear assumption implies that the internal current source is independent of the load impedance [32]. Shu and Lien [55] have proposed an improved analysis without this assumption to study the optimal AC/DC power generation for a rectified piezoelectric device. An accurate estimation of AC/DC power output is proposed explicitly and is related to electrically induced damping and energy conversion efficiency [56]. It is shown that the optimization criteria vary according to the relative strength of the electromechanical coupling. Other related studies can be found in [20, 24].

Recently, Guyomar et al [21] have developed a new electronic interface circuit to enhance power extraction. It is called synchronized switch harvesting on inductor (SSHI). This approach was derived from the so-called synchronized switch damping (SSD), a nonlinear technique developed originally by Richard et al [47] to investigate the effect of vibration damping on mechanical structures. It is shown that the electrical harvested power can be increased by as much as $400-900 \%$ over the standard technique [4, 5, 21, 39].
However, the approach they used to evaluate the performance of the SSHI circuit is based on the assumption that the periodic excitation and the speed of mass are in-phase. As a result, the effect of frequency deviation from resonance on the electrical behavior of an SSHI system is not included in their analysis. As vibration-based energy scavenging generators achieve the maximum power by requiring the matching of resonance frequency to applied frequency, power reduction due to frequency deviation cannot be ignored. Therefore, this paper revisits performance evaluation of the SSHI electronic interface based on the improved analysis [57]. A new estimate of average harvested power for the SSHI system is derived explicitly and validated numerically, and the connection between the standard and SSHI techniques is also established here. The electrical performance of the SSHI system is evaluated according to two key requirements: (1) the degradation in harvested power due to the non-perfect voltage inversion, and (2) the sensitivity in power deduction due to frequency deviation. The analysis reveals that the best use of the SSHI circuit is for systems in the midrange of electromechanical coupling, since the performance degradations are the least in these cases.

\section{Harvesting circuit: a standard interface}

Consider an energy conversion device including a vibrating piezoelectric structure together with an energy storage system. Suppose the modal density of such a device is widely separated and the structure is vibrating at around its resonance frequency; we may model the power generator as a mass + spring + damper + piezo structure $[21,42,48]$. The governing equations of this electromechanical model system can be described by

$$
\begin{aligned}
M \ddot{u}(t) & +\eta_{\mathrm{m}} \dot{u}(t)+K u(t)+\Theta V_{\mathrm{p}}(t)=F(t), \\
& -\Theta \dot{u}(t)+C_{p} \dot{V}_{\mathrm{p}}(t)=-I(t),
\end{aligned}
$$

where $u(t)$ is the displacement of the mass $M, V_{\mathrm{p}}(t)$ the voltage across the piezoelectric element, $\eta_{\mathrm{m}}$ the mechanical damping coefficient, $K$ the effective stiffness, $\Theta$ the effective piezoelectric coefficient, $C_{p}$ the clamped capacitance, $I(t)$ the current flowing into the specified circuit and $F(t)$ the external forcing function. These effective constants $M, K, \Theta$ and $C_{p}$ are dependent on the properties of the chosen materials and the specific types of piezoelectric generators. For example, we refer to [13] and [64] for the explicit expressions of these effective constants for the piezoelectric bimorph power generator. This paper considers a harmonic mechanical excitation given by

$$
F(t)=F_{0} \sin w t
$$

where $F_{0}$ is the constant magnitude and $w$ (in radians per second) is the angular frequency of vibration. This case is particularly chosen since many applications of piezoelectric materials for power generation involve the use of periodic straining of piezoelectric elements.

The unknowns in (1) and (2) are $u(t), V_{\mathrm{p}}(t)$ and $I(t)$, while there are only two equations. An additional equation is required and can be obtained from the consideration of the 


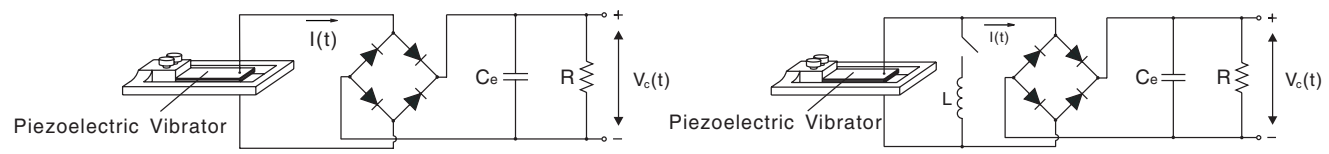

(a)

(b)

Figure 1. (a) A standard energy harvesting circuit. (b) An SSHI energy harvesting circuit.

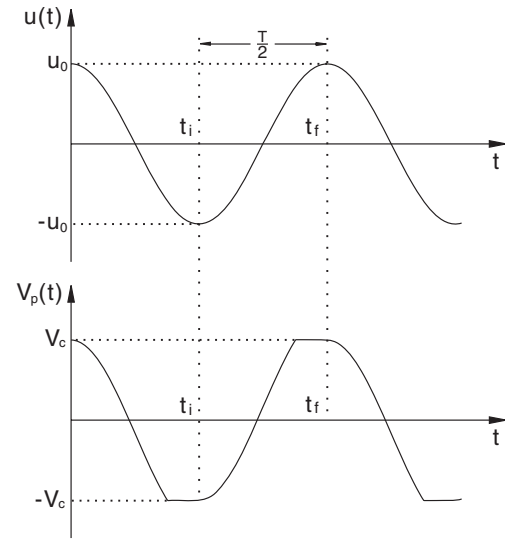

(a)

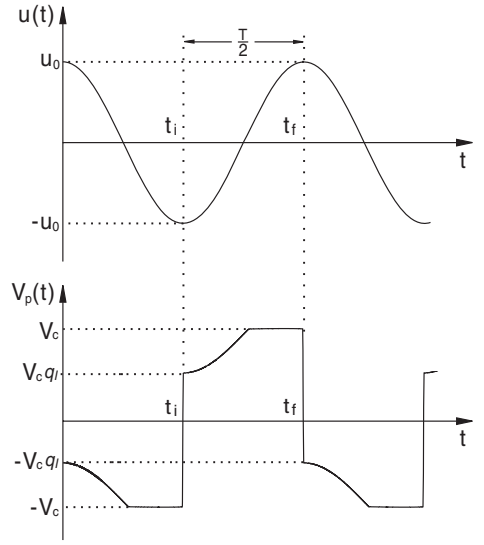

(b)

Figure 2. Typical waveforms of displacement and piezoelectric voltage for (a) the standard and for (b) the SSHI electronic interfaces.

chosen electronic interface between the piezoelectric element and the terminal electric load. Since the electronic load needs a stabilized DC voltage while a vibrating piezoelectric element generates an $\mathrm{AC}$ voltage, this requires a suitable AC-to-DC converter to ensure electrical compatibility. For example, figure 1(a) is a standard interface circuit commonly used for design analysis [42]. It provides an estimation of an upper bound of the real power that the piezoelectric power generator is able to deliver at a given excitation. The standard interface includes an AC/DC rectifier followed by a filtering capacitance $C_{e}$ added to smooth the DC rectified voltage $V_{\mathrm{c}}$. The terminal load is modeled using an equivalent resistor $R$ with power assumption equal to the average input power of the actual terminal electric load. Typically, the filter capacitor $C_{e}$ is chosen to be large enough so that the rectified voltage $V_{\mathrm{c}}$ is essentially constant to have a stable DC output voltage [42]. As a result, the rectified voltage $V_{\mathrm{c}}$ is independent of $C_{e}$ provided that the time constant $R C_{e}$ is much larger than the oscillating period of the generator [21].

To understand how the standard AC/DC electronic interface shown in figure 1(a) works for energy transfer, a perfect rectifying bridge is chosen for demonstration. It is open circuited if the piezoelectric voltage $\left|V_{\mathrm{p}}\right|<V_{\mathrm{c}}$, and the outgoing piezoelectric element current $I$ in (2) is null. On the other hand, when $\left|V_{\mathrm{p}}\right|$ reaches $V_{\mathrm{c}}$, the bridge conducts and the piezoelectric voltage is blocked at the rectified voltage; i.e., $\left|V_{\mathrm{p}}\right|=V_{\mathrm{c}}$. The conduction in the rectifier diodes is blocked again when $\left|V_{\mathrm{p}}\right|$ starts decreasing. Hence, the piezoelectric voltage $V_{\mathrm{p}}(t)$ either varies proportionally with the displacement $u(t)$ when the rectifying bridge is blocking, or is kept equal to $V_{\mathrm{c}}$ when the bridge conducts. As the model equations (1)-(3) are developed at the resonance mode of the device, a single-mode vibration of the structure is expected.
Thus, the displacement at the steady-state operation is assumed to be

$$
u(t)=u_{0} \sin (w t-\theta),
$$

where $u_{0}$ is the magnitude and $\theta$ is the phase shift. This assumption of choosing the sinusoidal form for displacement has been made by Guyomar et al [21] excluding the effect of the phase shift $\theta$. Shu and Lien [55] have included this effect and validated it both numerically and experimentally for the standard interface. The corresponding waveforms of $u(t)$ and $V_{\mathrm{p}}(t)$ are illustrated in figure 2(a).

To analyze the steady-state response of (1) and (2) connected to the standard AC/DC interface under the harmonic excitation (3), first note that it can be shown that the rectified voltage $V_{\mathrm{c}}$ is related to the magnitude of displacement $u_{0}$ by $[21,42,55]$

$$
V_{\mathrm{c}}=\frac{2 R \Theta w}{2 C_{p} R w+\pi} u_{0} .
$$

This formulation (5) can be derived by integrating (2) over a semi-period of vibration, and by using the fact that the average current flowing through the capacitance $C_{e}$ is null at steadystate operation. Thus, from (5), $u_{0}$ has to be determined to decide $V_{\mathrm{c}}$. There are three approaches in the recent literature for estimating it $[21,42,55]$. The first one models the piezoelectric device as the current source in parallel with its internal electrode capacitance $C_{p}[25,41,42]$. It is based on the assumption that the internal current source of the generator is independent of the external load impedance. However, the amplitude of the current source is closely related to that of displacement, which depends not only on the mechanical damping but also on the electrical damping at the resonant vibration $[36,56]$. This assumption is therefore not suitable when the effect of the electrical damping is significant. As a 
result, Guyomar et al [21] have proposed another estimation accounting for the effect of electromechanical coupling. Their estimation is based on the assumption that the external forcing function and the velocity of the mass are in-phase, or in other words the phase shift effect is neglected in (4). Instead, Shu and Lien [55] have included this phase factor in their improved analysis, and derived the analytic expressions of displacement magnitude $u_{0}$, rectified voltage $V_{\mathrm{c}}$ and harvested average power $P$. Their results are summarized as follows:

$$
\begin{aligned}
\bar{u}_{0}= & \frac{u_{0}}{\frac{F_{0}}{K}} \\
= & \frac{1}{\left\{\left(2 \zeta_{\mathrm{m}}+\frac{2 k_{\mathrm{e}}^{2} r}{\left(r \Omega+\frac{\pi}{2}\right)^{2}}\right)^{2} \Omega^{2}+\left(1-\Omega^{2}+\frac{k_{\mathrm{e}}^{2} r \Omega}{r \Omega+\frac{\pi}{2}}\right)^{2}\right\}^{\frac{1}{2}}}, \\
\bar{V}_{\mathrm{c}}= & \frac{V_{\mathrm{c}}}{\frac{F_{0}}{\Theta}}=\left(\frac{r \Omega}{r \Omega+\frac{\pi}{2}}\right) \\
& \times \frac{k_{\mathrm{e}}^{2}}{\left\{\left(2 \zeta_{\mathrm{m}}+\frac{2 k_{\mathrm{e}}^{2} r}{\left(r \Omega+\frac{\pi}{2}\right)^{2}}\right)^{2} \Omega^{2}+\left(1-\Omega^{2}+\frac{k_{\mathrm{e}}^{2} r \Omega}{r \Omega+\frac{\pi}{2}}\right)^{2}\right\}} \\
\bar{P}= & \frac{P}{\frac{1}{F_{0}^{2}}}=\frac{1}{\left(r \Omega+\frac{\pi}{2}\right)^{2}} \\
& \times \frac{k_{\mathrm{e}}^{2} \Omega^{2} r}{\left\{\left(2 \zeta_{\mathrm{m}}+\frac{2 k_{\mathrm{e}}^{2} r}{\left(r \Omega+\frac{\pi}{2}\right)^{2}}\right)^{2} \Omega^{2}+\left(1-\Omega^{2}+\frac{k_{\mathrm{e}}^{2} r \Omega}{r \Omega+\frac{\pi}{2}}\right)^{2}\right\}},
\end{aligned}
$$

where several non-dimensionless variables are introduced by

$$
\begin{gathered}
k_{\mathrm{e}}^{2}=\frac{\Theta^{2}}{K C_{p}}, \quad \zeta_{\mathrm{m}}=\frac{\eta_{\mathrm{m}}}{2 \sqrt{K M}}, \quad w_{\mathrm{sc}}=\sqrt{\frac{K}{M}}, \\
\Omega=\frac{w}{w_{\mathrm{sc}}}, \quad r=C_{p} w_{\mathrm{sc}} R .
\end{gathered}
$$

Above, $k_{\mathrm{e}}^{2}$ is the alternative electromechanical coupling coefficient, $\zeta_{\mathrm{m}}$ the mechanical damping ratio, $w_{\mathrm{sc}}$ the natural oscillation frequency (of the piezoelectric vibrator under the short circuit condition) and $\Omega$ and $r$ the normalized frequency and electric resistance. Notice that there are two resonances for the system since the piezoelectric structure exhibits both short circuit and open circuit stiffness. They are defined by

$$
\Omega_{\mathrm{sc}}=1, \quad \Omega_{\mathrm{oc}}=\sqrt{1+k_{\mathrm{e}}^{2}},
$$

where $\Omega_{\mathrm{sc}}$ and $\Omega_{\mathrm{oc}}$ are the frequency ratios of the short circuit and open circuit, respectively. Note that the shift in device natural frequency is pronounced if the coupling factor $k_{\mathrm{e}}^{2}$ is large.

The improved estimates (6)-(8) have been found to agree well with experimental observations and numerical simulations of (1) and (2) [55]. Therefore, these estimates are suitable for the electrical performance evaluation of the piezoelectric energy harvesting system embedded with the standard electronic interface. Moreover, if the vibration source is due to the periodic excitation of some base, this gives $F_{0}=$ $M A$, where $A$ is the magnitude of acceleration of the exciting base. From (8), the harvested average power per unit mass becomes

$$
\frac{P}{M}=\frac{A^{2}}{w_{\mathrm{sc}}} \bar{P}\left(r, \Omega, k_{\mathrm{e}}^{2}, \zeta_{\mathrm{m}}\right) .
$$

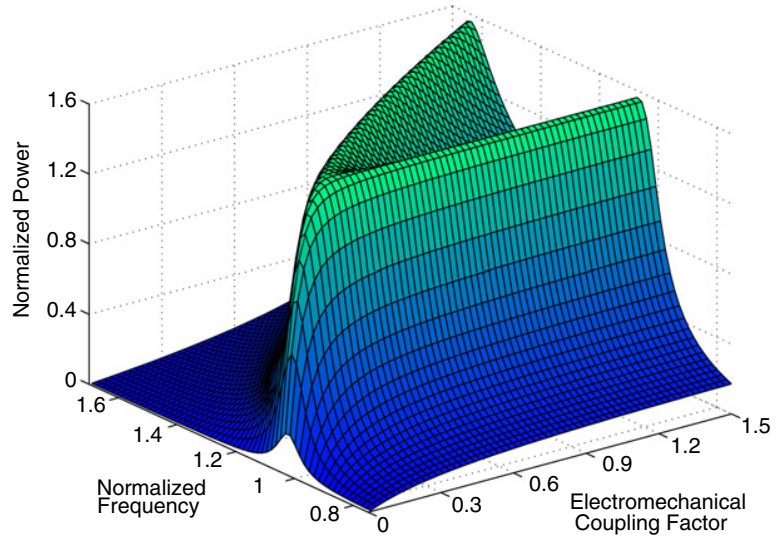

Figure 3. The normalized power $\bar{P}$ against the normalized frequency $\Omega$ and the electromechanical coupling factor $k_{\mathrm{e}}^{2}$ at the optimal conditions in the sense that $\bar{P}^{\text {opt }}\left(\Omega, k_{\mathrm{e}}^{2}, \zeta_{\mathrm{m}}\right)=\bar{P}\left(r^{\text {opt }}(\Omega), \Omega, k_{\mathrm{e}}^{2}, \zeta_{\mathrm{m}}\right)$ and $r^{\text {opt }}(\Omega)$ is determined by solving $\frac{\partial}{\partial r} \bar{P}\left(r, \Omega, k_{\mathrm{e}}^{2}, \zeta_{\mathrm{m}}\right)=0$. We use $\zeta_{\mathrm{m}}=0.04$ for the whole simulation. Notice that for large $k_{\mathrm{e}}^{2}$ there are two identical peaks of power evaluated at the frequency ratio close to $\Omega_{\mathrm{sc}}=1$ and $\Omega_{\mathrm{oc}}=\sqrt{1+k_{\mathrm{e}}^{2}}$. These peaks are saturated for much higher coupling factor $k_{\mathrm{e}}^{2} \gg 1$.

Thus, the optimization scheme is closely related to the tuning of the electric resistance, the selection of suitable operation points, and the magnitudes of the coupling coefficient and mechanical damping ratio. Basically, from (8) the harvested average power increases significantly for smaller mechanical damping ratio $\zeta_{\mathrm{m}}$ or larger electromechanical coupling coefficient $k_{\mathrm{e}}^{2}$. It is consistent with that found by Badel et al [3], who have performed an interesting experiment by comparing the performances of vibration-based piezoelectric power generators using a piezoelectric ceramic and a single crystal. Under the same operating conditions, the power generated using the single crystal is much higher than that using the ceramic, since according to their measurements the coupling factor $k_{\mathrm{e}}^{2}$ of the former is 20 times larger than that of the latter. However, one has to be cautious that the average harvested power approaches its saturation value for much larger $k_{\mathrm{e}}^{2}$, as illustrated in figure 3 .

The improved estimates have also been compared with the un-coupled and in-phase estimates according to the relative magnitudes of electromechanical coupling coefficient and mechanical damping ratio. The results of [55] show that the conventional un-coupled solution and in-phase estimate are suitable, provided that the ratio $\frac{k_{\mathrm{e}}^{2}}{\zeta_{\mathrm{m}}} \ll 1$, while the discrepancies among these distinct approaches become significant when $\frac{k_{\mathrm{e}}^{2}}{\zeta_{\mathrm{m}}}$ increases. If the shift in device natural frequency is pronounced and the mechanical damping ratio of the system is small, i.e. $\frac{k_{\mathrm{e}}^{2}}{\zeta_{\mathrm{m}}} \gg 1$, the harvested power is shown to have two optima evaluated at $\left(r_{1}^{\mathrm{opt}}, \Omega_{1}^{\mathrm{opt}}\right)$ and $\left(r_{2}^{\mathrm{opt}}, \Omega_{2}^{\mathrm{opt}}\right)$, where $\Omega_{1}^{\text {opt }}$ is close to $\Omega_{\mathrm{sc}}$ and the electric load $r_{1}^{\text {opt }}$ is very small, while $\Omega_{2}^{\mathrm{opt}}$ is close to $\Omega_{\mathrm{oc}}$ and $r_{2}^{\mathrm{opt}}$ is large. Indeed, table 1 summarizes the relation between the system parameters $k_{\mathrm{e}}^{2}$ and $\zeta_{\mathrm{m}}$ and the normalized load, displacement, voltage and power at these two optimal conditions. The first optimal pair is designed at the short circuit resonance $\Omega_{\mathrm{sc}}$ with the optimal load $r_{\mathrm{sc}}^{\mathrm{opt}} \propto \frac{1}{\frac{k_{\mathrm{e}}^{2}}{\xi_{\mathrm{m}}}}$, while the second one is designed at 


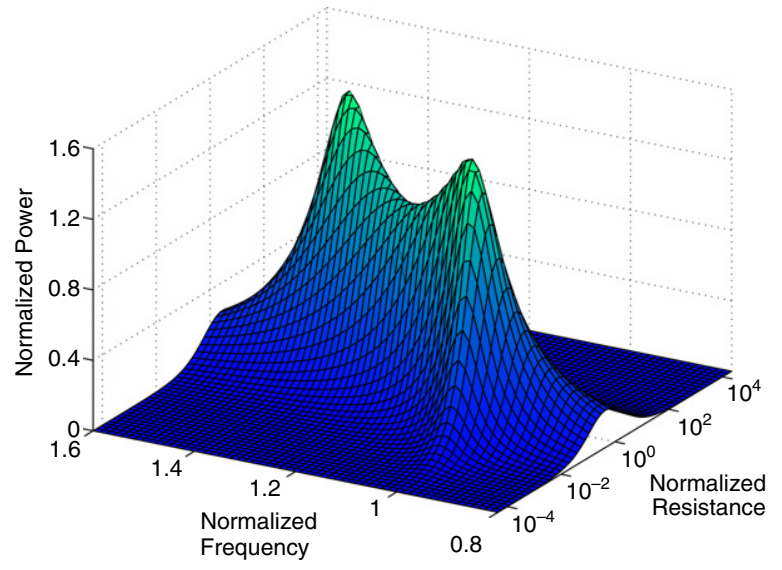

(a)

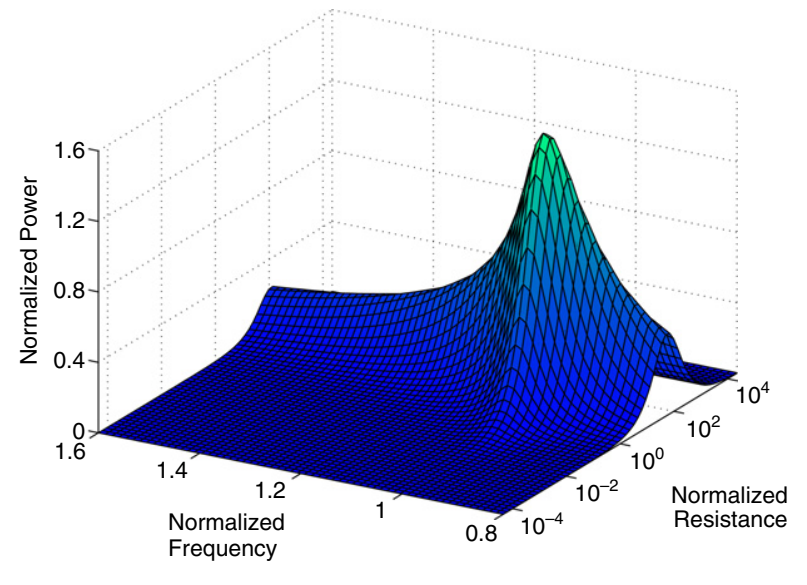

(b)

Figure 4. The normalized power against the normalized electric resistance and frequency ratio. (a) A strongly coupled electromechanical system using the standard AC/DC electronic interface $\left(k_{\mathrm{e}}^{2}=1.0, \zeta_{\mathrm{m}}=0.04, \frac{k_{\mathrm{e}}^{2}}{\zeta_{\mathrm{m}}}=25\right)$. (b) A weakly coupled electromechanical system using the ideal SSHI electronic interface $\left(k_{\mathrm{e}}^{2}=0.01, \zeta_{\mathrm{m}}=0.04, \frac{k_{\mathrm{e}}^{2}}{\zeta_{\mathrm{m}}}=0.25, Q_{\mathrm{I}}=\infty\right)$. Notice that (a) and (b) provide identical peaks of harvested power evaluated at different conditions.

Table 1. The relation between the system parameters $k_{\mathrm{e}}^{2}$ and $\zeta_{\mathrm{m}}$ and the normalized electric resistance, displacement, voltage and power operated at the short circuit $\left(\Omega_{\mathrm{sc}}\right)$ and open circuit $\left(\Omega_{\mathrm{oc}}\right)$ resonances [55]. Note that the condition $\frac{k_{\mathrm{e}}^{2}}{\zeta_{\mathrm{m}}} \gg 1$ is implied in the analysis.

\begin{tabular}{lll}
\hline Optimal conditions & $\Omega_{\mathrm{sc}}$ & $\Omega_{\mathrm{oc}}$ \\
\hline Resistance & $\left.r_{\mathrm{sc}}^{\mathrm{opt}} \propto \frac{1}{\frac{k_{\mathrm{e}}^{2}}{\zeta \mathrm{m}}}<r_{\mathrm{oc}}^{\mathrm{opt}} \propto \frac{1}{\left(1+k_{\mathrm{e}}^{2}\right.}\right) \frac{k_{\mathrm{e}}^{2}}{\zeta_{\mathrm{m}}}$ \\
Displacement & $\bar{u}_{0}^{\mathrm{opt}} \propto \frac{1}{\zeta_{\mathrm{m}}}>\bar{u}_{0}^{\mathrm{opt}} \propto \frac{1}{\zeta_{\mathrm{m}}\left(\sqrt{1+k_{\mathrm{e}}^{2}}\right)}$ \\
Voltage & $\bar{V}_{c}^{\mathrm{opt}} \propto 1<\bar{V}_{c}^{\mathrm{opt}} \propto \frac{1}{\sqrt{1+k_{\mathrm{e}}^{2}}} \frac{k_{\mathrm{e}}^{2}}{\zeta_{\mathrm{m}}}$ \\
Power & $\bar{P}^{\mathrm{opt}} \propto \frac{1}{\zeta_{\mathrm{m}}}=\bar{P}^{\mathrm{opt}} \propto \frac{1}{\zeta_{\mathrm{m}}}$ \\
\hline
\end{tabular}

the open circuit resonance $\Omega_{\mathrm{oc}}$ with the optimal load $r_{\mathrm{oc}}^{\mathrm{opt}} \propto$ $\frac{1}{\left(1+k^{2}\right)} \frac{k_{\mathrm{e}}^{2}}{\zeta_{\mathrm{m}}}$. They give identical values of maximum harvested power, which depends only on the mechanical damping ratio $\zeta_{\mathrm{m}}$. Unlike the power, the displacement is higher at $\Omega_{\mathrm{sc}}$ than at $\Omega_{\mathrm{oc}}$, while the voltage operating at the first peak is one order of magnitude smaller than that operating at the second peak.

Finally, figure 4(a) gives the dependence of the normalized harvested power on the normalized resistance and frequency ratio for the case of strong electromechanical coupling. While such a strong coupling is not commonly observed in the conventional piezoelectric power generators, we particularly emphasize it here since we will show in the next section that the behavior of an ideal SSHI system is similar to that of a strongly coupled electromechanical standard system excited at around the short circuit resonance. This finding is generally valid no matter whether the real electromechanical system is weakly or strongly coupled.

\section{Harvesting circuit: an SSHI interface}

An SSHI electronic interface consists of adding a switch and an inductance $L$ connected in series and is in parallel with the piezoelectric element as shown in figure 1(b). The electronic switch is triggered according to the maximum and minimum of the displacement of the mass, causing the processing of piezoelectric voltage to be synchronized with the extreme values of displacement.

To illustrate the electrical behavior of this nonlinear processing circuit, consider the harmonic excitation given by (3). In view of the single-mode excitation, the mechanical displacement $u(t)$ is assumed to be sinusoidal as in (4) in steady-state operation. The validation of this assumption will be examined in section 4.1 by considering the output voltage. The waveform of the piezoelectric voltage $V_{\mathrm{p}}(t)$, however, may not be sinusoidal and is dependent on the specific type of the interface circuit connected to the piezoelectric element. To see this, let $T=\frac{2 \pi}{w}$ be the period of mechanical excitation and $t_{i}$ and $t_{f}$ be two time instants such that the displacement $u(t)$ undergoes from the minimum $-u_{0}$ to the maximum $u_{0}$ as illustrated in figure 2(b). The switch is turned off most of the time during this semi-period $\left(t_{i}^{+}, t_{f}\right)$. When it is turned on at the time instant $t_{i},\left|V_{\mathrm{p}}(t)\right|$ remains lower than the rectified voltage $V_{c}$. So the rectifying bridge is open circuited, and an oscillating electrical circuit composed by the inductance $L$ and the piezoelectric capacitance $C_{p}$ is established, giving rise to an inversion process for the piezoelectric voltage $V_{\mathrm{p}}$. Specifically, let $\Delta t$ be the half electric period of this oscillating $L-C_{p}$ circuit. It is equal to [21]

$$
\Delta t=\pi \sqrt{L C_{p}} .
$$

We assume that the inversion process is quasi-instantaneous in the sense that the inversion time is chosen to be much smaller than the period of the mechanical vibration; i.e., $\Delta t=$ $t_{i}^{+}-t_{i} \ll T$. The switch is kept closed during this small time period $\Delta t$, resulting in the reverse of voltage on the piezoelectric element, i.e.,

$$
V_{\mathrm{p}}\left(t_{i}^{+}\right)=-V_{\mathrm{p}}\left(t_{i}\right) \mathrm{e}^{\frac{-\pi}{2 Q_{\mathrm{I}}}}=V_{\mathrm{c}} q_{\mathrm{I}}, \quad q_{\mathrm{I}}=\mathrm{e}^{\frac{-\pi}{2 Q_{\mathrm{I}}}}
$$


as illustrated in figure 2(b). Above, $Q_{\mathrm{I}}$ is the inversion quality factor due to the energy loss mainly from the inductor in series with the switch. As a result, the current outgoing from the piezoelectric element through the rectifier during a half vibration period can be obtained by integrating (2) from time $t_{i}^{+}$to $t_{f}[3]$

$$
\begin{gathered}
\int_{t_{i}^{+}}^{t_{f}}\left\{-\Theta \dot{u}(t)+C_{p} \dot{V}_{\mathrm{p}}(t)\right\} \mathrm{d} t=-2 \Theta u_{0} \\
+C_{p}\left(1-\mathrm{e}^{-\frac{\pi}{2 Q_{\mathrm{I}}}}\right) V_{\mathrm{c}}=-\frac{T}{2} \frac{V_{\mathrm{c}}}{R}
\end{gathered}
$$

since the rectifier bridge is blocking during the inversion process and the inversion time $\Delta t \ll T$. The relation between the magnitude of displacement $u_{0}$ and the rectified voltage $V_{\mathrm{c}}$ is therefore obtained by

$$
V_{\mathrm{c}}=\frac{2 R \Theta w}{\left(1-q_{\mathrm{I}}\right) C_{p} R w+\pi} u_{0} .
$$

The rest of the problem is to estimate the magnitude of displacement $u_{0}$ and the phase shift $\theta$. To solve them, consider the balance of energy first. Let (1) be multiplied by $\dot{u}(t)$ and (2) be multiplied by $V_{\mathrm{p}}(t)$. Integration of the addition of these two equations from time $t_{i}^{+}$to $t_{f}$ gives the equation of the energy balance

$$
\begin{aligned}
& \int_{t_{i}^{+}}^{t_{f}} F(t) \dot{u}(t) \mathrm{d} t=\int_{t_{i}^{+}}^{t_{f}} \eta_{\mathrm{m}} \dot{u}^{2}(t) \mathrm{d} t+\int_{t_{i}^{+}}^{t_{f}} V_{\mathrm{p}}(t) I(t) \mathrm{d} t \\
& \quad+\left.\frac{1}{2} M \dot{u}^{2}(t)\right|_{t_{i}^{+}} ^{t_{f}}+\left.\frac{1}{2} K u^{2}(t)\right|_{t_{i}^{+}} ^{t_{f}}+\left.\frac{1}{2} C_{p} V_{\mathrm{p}}^{2}(t)\right|_{t_{i}^{+}} ^{t_{f}} \\
& =\int_{t_{i}^{+}}^{t_{f}} \eta_{\mathrm{m}} \dot{u}^{2}(t) \mathrm{d} t+\int_{t_{i}^{+}}^{t_{f}} V_{\mathrm{p}}(t) I(t) \mathrm{d} t+\frac{1}{2} C_{p}\left(1-q_{\mathrm{I}}^{2}\right) V_{\mathrm{c}}^{2}
\end{aligned}
$$

where

$$
\begin{gathered}
\int_{t_{i}^{+}}^{t_{f}} F(t) \dot{u}(t) \mathrm{d} t=\frac{\pi}{2} F_{0} u_{0} \sin \theta, \\
\int_{t_{i}^{+}}^{t_{f}} \eta_{\mathrm{m}} \dot{u}^{2}(t) \mathrm{d} t=\frac{\pi}{2} \eta_{\mathrm{m}} w u_{0}^{2}, \\
\int_{t_{i}^{+}}^{t_{f}} V_{\mathrm{p}}(t) I(t) \mathrm{d} t=\frac{\pi}{w} \frac{V_{\mathrm{c}}^{2}}{R} .
\end{gathered}
$$

There are now three unknowns, $u_{0}, \theta$ and $V_{\mathrm{c}}$, while only two equations, (12) and (13), are found. The third equation connecting these unknowns can be obtained as follows. Notice that from (2)

$$
\Theta \dot{V}_{\mathrm{p}}(t)=\frac{\Theta}{C_{p}}[-I(t)+\Theta \dot{u}(t)] .
$$

Differentiating (1) with respect to time $t$ and substituting (14) into it, we find

$$
\begin{aligned}
& M \frac{\mathrm{d}}{\mathrm{d} t} \ddot{u}(t)+\eta_{\mathrm{m}} \frac{\mathrm{d}}{\mathrm{d} t} \dot{u}(t)+\left(K+\frac{\Theta^{2}}{C_{p}}\right) \frac{\mathrm{d}}{\mathrm{d} t} u(t)-\frac{\Theta}{C_{p}} I(t) \\
& =\frac{\mathrm{d}}{\mathrm{d} t} F(t) .
\end{aligned}
$$

Integrating (15) with respect to time $t$ from $t_{i}^{+}$to $t_{f}$ and using (4) provides the third equation

$$
\left(K-M w^{2}+\frac{\Theta^{2}}{C_{p}}\right) u_{0}-\frac{\pi \Theta}{2 C_{p} w R} V_{\mathrm{c}}=F_{0} \cos \theta .
$$

Thus, the unknown $\theta$ can be eliminated from (13) and (16). This gives

$$
\begin{aligned}
F_{0}^{2} & =\left\{\eta_{\mathrm{m}} w u_{0}+\left(\frac{2}{w R}+\left(1-q_{\mathrm{I}}^{2}\right) \frac{C_{p}}{\pi}\right) \frac{V_{\mathrm{c}}^{2}}{u_{0}}\right\}^{2} \\
& +\left\{\left(K-M w^{2}+\frac{\Theta^{2}}{C_{p}}\right) u_{0}-\frac{\pi \Theta}{2 C_{p} w R} V_{\mathrm{c}}\right\}^{2} .
\end{aligned}
$$

The above equation (17) can be further simplified to find $u_{0}$ by using the relation between $u_{0}$ and $V_{\mathrm{c}}$ given by (12), and this gives

$$
\begin{aligned}
& u_{0}= \\
& \frac{F_{0}}{\left\{\left(\eta_{\mathrm{m}}+\frac{2\left[1+\frac{C_{p} R w}{2 \pi}\left(1-q_{1}^{2}\right)\right] \Theta^{2} R}{\left(\frac{\left(1-q_{1}\right)}{2} C_{p} w R+\frac{\pi}{2}\right)^{2}}\right)^{2} w^{2}+\left(K-w^{2} M+\frac{\frac{\left(1-q_{\mathrm{I}}\right)}{2} w \Theta^{2} R}{\frac{\left(1-q_{q_{1}}\right)}{2} C_{p} w R+\frac{\pi}{2}}\right)^{2}\right\}^{\frac{1}{2}}} .
\end{aligned}
$$

The average harvested power is therefore obtained once $u_{0}$ is determined since from (12)

$$
P=\frac{V_{\mathrm{c}}^{2}}{R}=\frac{4 R \Theta^{2} w^{2}}{\left\{\left(1-q_{\mathrm{I}}\right) C_{p} R w+\pi\right\}^{2}} u_{0}^{2} .
$$

The following summarizes our main results for the piezoelectric element connected to the SSHI interface circuit. The normalized displacement magnitude $\bar{u}_{0}^{\mathrm{SSHI}}$, rectified voltage $\bar{V}_{\mathrm{c}}^{\mathrm{SSH}}$ and average harvested power $\bar{P}^{\mathrm{SSHI}}$ are given respectively by

$\bar{u}_{0}^{\mathrm{SSHI}}=\frac{u_{0}^{\mathrm{SSHI}}}{\frac{F_{0}}{K}}=$

$$
\overline{\left\{\left(2 \zeta_{\mathrm{m}}+\frac{2\left[1+\frac{r \Omega}{2 \pi}\left(1-q_{\mathrm{I}}^{2}\right)\right] k_{\mathrm{e}}^{2} r}{\left(\frac{\left(1-q_{\mathrm{I}}\right)}{2} r \Omega+\frac{\pi}{2}\right)^{2}}\right)^{2} \Omega^{2}+\left(1-\Omega^{2}+\frac{\frac{\left(1-q_{\mathrm{I}}\right)}{2} k_{\mathrm{e}}^{2} r \Omega}{\frac{\left(1-q_{\mathrm{I}}\right)}{2} r \Omega+\frac{\pi}{2}}\right)^{2}\right\}^{\frac{1}{2}}},
$$$$
\bar{V}_{\mathrm{c}}^{\mathrm{SSHI}}=\frac{V_{\mathrm{c}}^{\mathrm{SSHI}}}{\frac{F_{0}}{\Theta}}=\left(\frac{r \Omega}{\frac{\left(1-q_{\mathrm{I}}\right)}{2} r \Omega+\frac{\pi}{2}}\right)
$$

$$
\times \frac{k_{\mathrm{e}}^{2}}{\left\{\left(2 \zeta_{\mathrm{m}}+\frac{2\left[1+\frac{r \Omega}{2 \pi}\left(1-q_{\mathrm{I}}^{2}\right)\right] k_{\mathrm{e}}^{2} r}{\left(\frac{\left(1-q_{\mathrm{I}}\right)}{2} r \Omega+\frac{\pi}{2}\right)^{2}}\right)^{2} \Omega^{2}+\left(1-\Omega^{2}+\frac{\frac{\left(1-q_{\mathrm{I}}\right)}{2} k_{\mathrm{e}}^{2} r \Omega}{\frac{\left(1-q_{\mathrm{I}}\right)}{2} r \Omega+\frac{\pi}{2}}\right)^{2}\right\}^{\frac{1}{2}}},
$$

$$
\bar{P}^{\mathrm{SSHI}}=\frac{P^{\mathrm{SSHI}}}{\frac{F_{0}^{2}}{w_{\mathrm{sc}} M}}=\left(\frac{1}{\frac{\left(1-q_{\mathrm{I}}\right)}{2} r \Omega+\frac{\pi}{2}}\right)^{2}
$$

$$
\times \frac{k_{\mathrm{e}}^{2} \Omega^{2} r}{\left(2 \zeta_{\mathrm{m}}+\frac{2\left[1+\frac{r \Omega}{2 \pi}\left(1-q_{1}^{2}\right)\right] k_{\mathrm{e}}^{2} r}{\left(\frac{\left(1-q_{\mathrm{I}}\right)}{2} r \Omega+\frac{\pi}{2}\right)^{2}}\right)^{2} \Omega^{2}+\left(1-\Omega^{2}+\frac{\frac{\left(1-q_{1}\right)}{2} k_{\mathrm{e}}^{2} r \Omega}{\frac{\left(1-q_{1}\right.}{2} r \Omega+\frac{\pi}{2}}\right)^{2}} .
$$

All above are expressed in terms of non-dimensionless parameters defined in (9).

Finally, Guyomar et al [21] have used the in-phase assumption to analyze the electrical performance of the power generator using the SSHI interface. To be precise, they have assumed that the external forcing function and the velocity of 


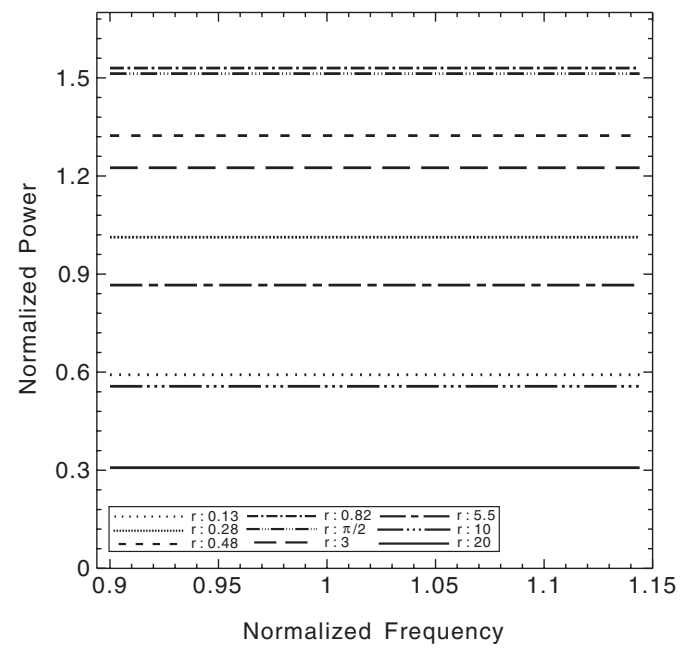

(a)

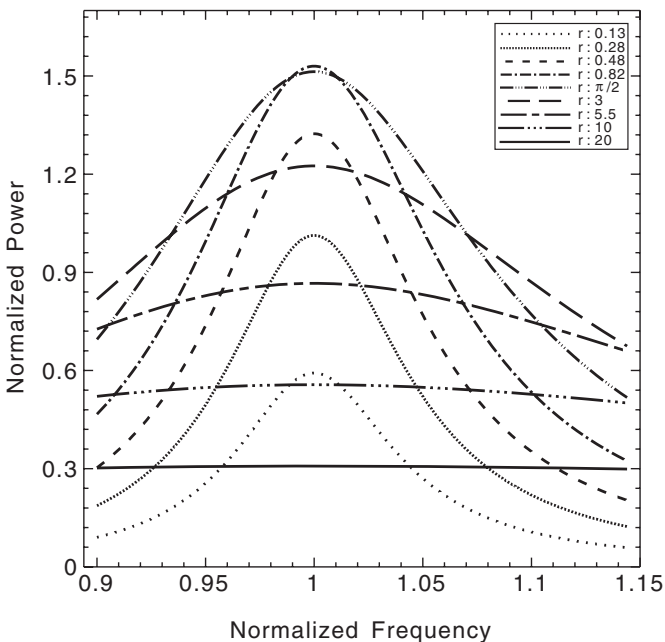

(b)

Figure 5. Normalized harvested power versus frequency ratio for various load resistances based on (a) the in-phase estimate $\bar{P}_{\text {in-phase }}^{\mathrm{SSHI}}$ and (b) the improved analytic estimate $\bar{P}^{\text {SSHI }}$. We take $k_{\mathrm{e}}^{2}=0.09, \zeta_{\mathrm{m}}=0.04$ and $Q_{\mathrm{I}}=\infty$ for the whole simulation.

the mass are in-phase, giving rise to no phase shift effect in their formulation. The following summarizes their results for future comparisons:

$$
\begin{aligned}
& \bar{u}_{\text {in }- \text { phase }}^{\text {SSHI }}=\frac{u_{\text {in }- \text { phase }}^{\text {SSHI }}}{\frac{F_{0}}{K}}=\frac{1}{\left\{2 \zeta_{\mathrm{m}}+\frac{2\left[1+\frac{r \Omega}{2 \pi}\left(1-q_{\mathrm{I}}^{2}\right)\right] k_{\mathrm{e}}^{2} r}{\left(\frac{\left(1-q_{\mathrm{I}}\right)}{2} r \Omega+\frac{\pi}{2}\right)^{2}}\right\} \Omega}, \\
& \bar{V}_{\text {in }- \text { phase }}^{\text {SSHI }}=\frac{V_{\text {in-phase }}^{\text {SSHI }}}{\frac{F_{0}}{\Theta}}=\left(\frac{r}{\frac{\left(1-q_{\mathrm{I}}\right)}{2} r \Omega+\frac{\pi}{2}}\right) \\
& \times \frac{k_{\mathrm{e}}^{2}}{\left\{2 \zeta_{\mathrm{m}}+\frac{2\left[1+\frac{r \Omega}{2 \pi}\left(1-q_{\mathrm{I}}^{2}\right)\right] k_{\mathrm{e}}^{2} r}{\left(\frac{\left(1-q_{\mathrm{I}}\right)}{2} r \Omega+\frac{\pi}{2}\right)^{2}}\right\}}, \\
& \bar{P}_{\text {in }- \text { phase }}^{\text {SSHI }}=\frac{P_{\text {in-phase }}^{\text {SSHI }}}{\frac{F_{0}^{2}}{w_{\mathrm{sc}} M}}=\frac{1}{\left(\frac{\left(1-q_{\mathrm{I}}\right)}{2} r \Omega+\frac{\pi}{2}\right)^{2}} \\
& \times \frac{k_{\mathrm{e}}^{2} r}{\left\{2 \zeta_{\mathrm{m}}+\frac{2\left[1+\frac{r \Omega}{2 \pi}\left(1-q_{\mathrm{I}}^{2}\right)\right] k_{\mathrm{e}}^{2} r}{\left(\frac{\left(1-q_{1}\right)}{2} r \Omega+\frac{\pi}{2}\right)^{2}}\right\}^{2}} .
\end{aligned}
$$

\section{Discussion}

\subsection{In-phase versus improved analysis}

The in-phase estimate shows a lack of frequency dependence. To see this, we consider a case where the ratio of the electromechanical coupling factor to the mechanical damping ratio is in the medium range; i.e., $\frac{k_{\mathrm{e}}^{2}}{\zeta_{\mathrm{m}}}$ is of order one. We take $k_{\mathrm{e}}^{2}=0.09$ and $\zeta_{\mathrm{m}}=0.04$, and therefore $\frac{k_{\mathrm{e}}^{2}}{\zeta_{\mathrm{m}}}=2.5$. It can be shown that other parameters provide similar contrasting comparisons between the in-phase and improved estimates. Figure 5(a) describes the average harvested power versus frequency ratio for various load resistances based on the inphase estimate while figure 5(b) is based on our improved analysis. For fair comparison, the ideal inversion of voltage is implied in this case; i.e., $Q_{\mathrm{I}}=\infty$. We will discuss this condition in more detail later. Clearly, the two estimates predict identical values of the optimal power. However, the in-phase estimate is unable to predict the system behavior when the applied driving frequency deviates from the system resonance frequency. As the reduction in power is significant due to frequency deviation, such an effect cannot be ignored in practical design.

We next validate our improved estimates numerically by transforming (1)-(3) to an equivalent circuit with $R^{*}=\frac{\eta_{\mathrm{m}}}{\Theta^{2}}$ as resistance, $L^{*}=\frac{M}{\Theta^{2}}$ as inductance and $C^{*}=\frac{\Theta^{2}}{K}$ as capacitance as shown in figure 6(a). We use the software PSpice to simulate this equivalent circuit connected to the SSHI interface. We take $k_{\mathrm{e}}^{2}=0.09, \zeta_{\mathrm{m}}=0.04$ and consider the non-ideal voltage inversion with quality factor $Q_{\mathrm{I}}=$ 2.6 [21]. The results are illustrated in figure 6(b), where the normalized power is plotted against the frequency ratio evaluated at the optimal electric load, $r^{\text {opt }}=1.01$ in this case. The predicted results from the in-phase and analytic estimates are represented by dashed and solid lines in figure 6(b), while the numerical results are marked using open circles there. Apparently, the numerical simulation favors results predicted based on our analytic improved estimate. Therefore, from now on, we will use (19)-(21) as the fundamental tool for the performance evaluation of the piezoelectric energy harvesting system embedded with an SSHI interface circuit.

\subsection{Ideal inversion of piezoelectric voltage}

To see how the SSHI electronic interface boosts power extraction, consider an ideal case where the inversion of the piezoelectric voltage $V_{\mathrm{p}}$ is complete; i.e., $Q_{\mathrm{I}}=\infty$. From (11) this gives $q_{\mathrm{I}}=1$ and the normalized harvested power from (21) 


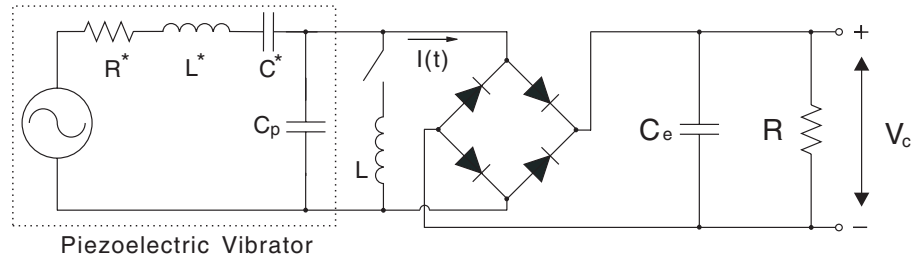

(a)

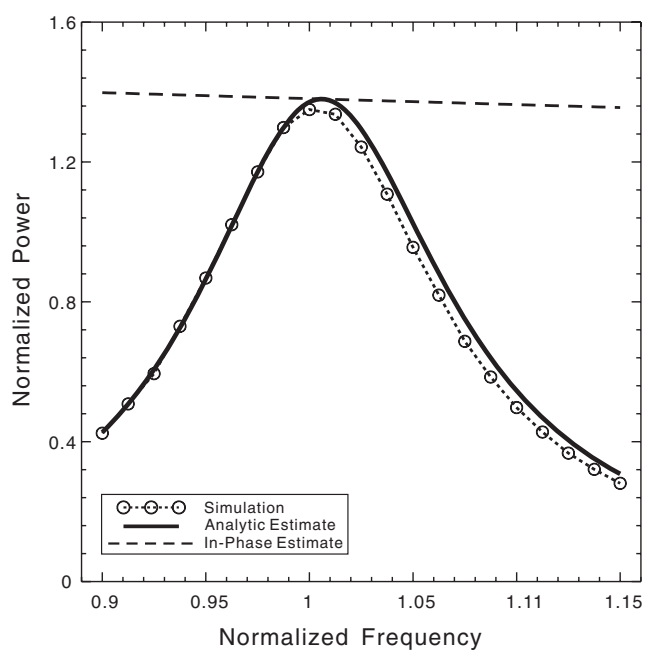

(b)

Figure 6. Numerical validation with parameters $k_{\mathrm{e}}^{2}=0.09, \zeta_{\mathrm{m}}=0.04$ and $Q_{\mathrm{I}}=2.6$. (a) The equivalent circuit model for the system of equations (1)-(3) connected to the SSHI interface. (b) The simulation results compared with those predicted by analytic and in-phase estimates.

becomes

$$
\bar{P}^{\text {SSHI }}=\frac{4}{\pi^{2}} \frac{r k_{\mathrm{e}}^{2} \Omega^{2}}{\left\{4\left(\zeta_{\mathrm{m}}+\frac{4 k_{\mathrm{e}}^{2} r}{\pi^{2}}\right)^{2} \Omega^{2}+\left(1-\Omega^{2}\right)^{2}\right\}} .
$$

The optimal electric load resistance and the normalized power operated at $\Omega_{\mathrm{sc}}$ are therefore

$$
r^{\mathrm{opt}}=\frac{\pi^{2}}{4} \frac{1}{\frac{k_{\mathrm{e}}^{2}}{\zeta_{\mathrm{m}}}},\left.\quad \bar{P}^{\mathrm{SSHI}}\right|_{r=r^{\mathrm{opt}, \Omega=1}}=\frac{1}{16 \zeta_{\mathrm{m}}} .
$$

From (26), the optimal load resistance is inversely proportional to the ratio $\frac{k_{\mathrm{e}}^{2}}{\zeta_{\mathrm{m}}}$ while the corresponding optimal power depends only on the mechanical damping ratio $\zeta_{\mathrm{m}}$ and is independent of the electromechanical coupling coefficient $k_{\mathrm{e}}^{2}$. Comparing all of these features with table 1 suggests that the behavior of the power harvesting system using the SSHI interface is similar to that of a strongly coupled electromechanical system using the standard interface and operated at the short circuit resonance $\Omega_{\mathrm{sc}}$. Indeed, comparing (5) with (12) suggests that the original capacitance $C_{p}$ is replaced by the effective capacitance $\bar{C}_{p}=$ $\frac{\left(1-q_{\mathrm{I}}\right)}{2} C_{p}$. Therefore, the effective electromechanical coupling coefficient $\bar{k}_{\mathrm{e}}^{2}$ can be realized by

$$
\bar{k}_{\mathrm{e}}^{2}=\frac{\Theta^{2}}{K \bar{C}_{p}}=\frac{2 \Theta^{2}}{K\left(1-q_{\mathrm{I}}\right) C_{p}} .
$$

For the ideal inversion, $q_{\mathrm{I}} \rightarrow 1$, and this gives $\frac{\bar{k}_{\mathrm{e}}^{2}}{\bar{\zeta}_{\mathrm{m}}} \rightarrow \infty$ no matter what the original value of the ratio $\frac{k_{\mathrm{e}}^{2}}{\zeta_{\mathrm{m}}}$ is. The response using the SSHI interface is therefore similar to that using the standard interface in a strongly coupled electromechanical system operated at $\Omega_{\mathrm{sc}}$. In addition, according to table 1 , there exists another identical peak of power operated at the open circuit resonance. But now this frequency ratio is realized as $\bar{\Omega}_{\mathrm{oc}}=\sqrt{1+\bar{k}_{\mathrm{e}}^{2}} \rightarrow \infty$. Hence, the second peak of power is moved to the infinite point in the $(r, \Omega)$ space, and therefore there is only one peak of power for the SSHI electronic interface, no matter whether the real electromechanical system is weakly or strongly coupled, as schematically shown in figure $4(\mathrm{~b})$.

In addition, we particularly take $k_{\mathrm{e}}^{2}=0.01$ and $\zeta_{\mathrm{m}}=0.04$ in figure 4(b) so that the electromechanical generator itself is weakly coupled $\left(\frac{k_{\mathrm{e}}^{2}}{\zeta_{\mathrm{m}}}=0.25\right)$. The harvested power obtained using the standard harvesting circuit is pretty small in this case, since it has been shown that [55]

$$
\begin{aligned}
& \bar{P}\left(r^{\mathrm{opt}}=\frac{\pi}{2}, \Omega=1, k_{\mathrm{e}}^{2}, \zeta_{\mathrm{m}}\right) \\
& \quad \approx\left(\frac{2}{\pi} \frac{k_{\mathrm{e}}^{2}}{\zeta_{\mathrm{m}}}\right) \frac{1}{16 \zeta_{\mathrm{m}}} \ll \frac{1}{16 \zeta_{\mathrm{m}}}=\left(\bar{P}^{\mathrm{SSHI}}\right)_{\max }
\end{aligned}
$$

if $\frac{k_{\mathrm{e}}^{2}}{\zeta_{\mathrm{m}}} \ll 1$. But the inclusion of the SSHI circuit boosts the average harvested power, whose maximum is the same as that using a strongly coupled electromechanical generator connected to the standard interface as illustrated in figure 4(a) 
$\left(k_{\mathrm{e}}^{2}=1.0, \zeta_{\mathrm{m}}=0.04\right.$ and $\left.\frac{k_{\mathrm{e}}^{2}}{\zeta_{\mathrm{m}}}=25\right)$. Therefore, the harvested power increases tremendously for any weak coupling SSHI system at the cost of using a much larger optimal electric load, which is proportional to $\frac{1}{\frac{k_{\mathrm{e}}^{2}}{\xi_{\mathrm{m}}}}$ according to (26).

\section{Comparisons}

We now compare the electrical performance of a vibrationbased piezoelectric power generator using the standard and SSHI electronic interfaces according to the different ratios of $\frac{k_{\mathrm{e}}^{2}}{\zeta_{\mathrm{m}}}$. As in many practical situations, the inversion of the piezoelectric voltage $V_{\mathrm{p}}$ is not perfect $\left(Q_{\mathrm{I}} \neq \infty\right)$, which accounts for a certain amount of the performance degradation using the SSHI electronic interface. We take $Q_{\mathrm{I}}=2.6$ for comparison here [21]. It is possible to have a larger value of quality factor $Q_{\mathrm{I}}$ by requiring the use of a low loss inductor [33].

First, consider a weakly coupled electromechanical system; i.e., the ratio $\frac{k_{\mathrm{e}}^{2}}{\zeta_{\mathrm{m}}} \ll 1$. We take $k_{\mathrm{e}}^{2}=0.01$ and $\zeta_{\mathrm{m}}=0.04$ for demonstration. This gives $\frac{k_{\mathrm{e}}^{2}}{\zeta_{\mathrm{m}}}=$ 0.25 . The harvested power versus frequency ratio for various normalized resistances is shown in figure 7(a) based on the standard interface and in figure 7(d) based on the SSHI interface. The maximum normalized power generated for the ideal voltage inversion is around $\left.\bar{P}^{\text {SSHI }}\right|_{Q_{1}=\infty}=1.56$, while $\left.\bar{P}^{\text {SSHI }}\right|_{Q_{\mathrm{I}}=2.6}=0.67$ in the non-ideal case. In spite of the significant performance degradation in this case, the achieved optimal power is three times larger than that using the standard interface $(\bar{P}=0.23)$ at the cost of using a larger matching load resistance from $r^{\mathrm{opt}}=\pi / 2$ to 5.2 by comparing figure 7 (a) with figure $7(\mathrm{~d})$.

Next, suppose the electromechanical coupling is in the medium range; i.e., the ratio of $\frac{k_{\mathrm{e}}^{2}}{\zeta_{\mathrm{m}}}$ is of order one. We take $k_{\mathrm{e}}^{2}=0.09$ and $\zeta_{\mathrm{m}}=0.04$. This gives $\frac{k_{\mathrm{e}}^{2}}{\zeta_{\mathrm{m}}}=2.25$. The harvested power versus frequency ratio for various normalized resistances is shown in figure 7(b) based on the standard interface and in figure 7(e) based on the SSHI interface. The maximum normalized power for the non-ideal voltage inversion is $\left.\bar{P}^{\mathrm{SSHI}}\right|_{Q_{\mathrm{I}}=2.6}=1.38$, which is slightly smaller than the ideal case $\left(\left.\bar{P}^{\mathrm{SSHI}}\right|_{Q_{\mathrm{I}}=\infty}=1.56\right)$, but it is slightly larger than that using the standard electronic interface $(\bar{P}=$ 1.20). While there is no significant increase of power output using the SSHI electronic interface in this case, figure 7(e) demonstrates that the harvested power evaluated at around the optimal load is less sensitive to frequency deviating from the resonant vibration. For example, the amount of normalized harvested power $\bar{P}$ evaluated at $r=\frac{\pi}{2}$ in the standard case drops from 1.2 to 0.6 for about $5 \%$ frequency deviation, and from 1.2 to 0.2 for about $10 \%$ frequency deviation. However, under the same conditions, the normalized harvested power $\bar{P}^{\text {SSHI }}$ in the SSHI circuit drops from 1.3 to only 1.0 for about $5 \%$ frequency deviation, and from 1.3 to 0.5 for about $10 \%$ frequency deviation. This frequency-insensitive feature is much more pronounced in the case of ideal voltage inversion, as can be seen by comparing figure 5(b) with figure 7(b).

Finally, we turn to a strongly coupled electromechanical system $\left(\frac{k_{\mathrm{e}}^{2}}{\zeta_{\mathrm{m}}} \gg 1\right)$. Our numerous numerical simulations show that the rule of thumb for the appearance of double identical peaks of power in the standard case is when

$$
\frac{k_{\mathrm{e}}^{2}}{\zeta_{\mathrm{m}}} \geqslant 10 .
$$

We then take $k_{\mathrm{e}}^{2}=1.0$ and $\zeta_{\mathrm{m}}=0.04$, and this gives $\frac{k_{\mathrm{e}}^{2}}{\zeta_{\mathrm{m}}}=25$. The harvested power versus frequency ratio for various normalized resistances is shown in figure 7(c) based on the standard interface and in figure 7(f) based on the SSHI interface. In the standard case, the harvested power has two identical optimal peaks, and the switching between these two peaks can be achieved by varying the electric loads. The envelope of these peaks has a local minimum, which is closely related to the minimum proof mass displacement. On the other hand, there is only one peak of power in the SSHI circuit, as explained in the previous section. Unlike the standard case as illustrated in figure 7(c), the peaks of the average harvested power decrease significantly as the load resistances increase, as shown in figure 7(f). In addition, it can be seen from (26) that the optimal electric load for the SSHI system is very small, since $\frac{k_{\mathrm{e}}^{2}}{\zeta_{\mathrm{m}}} \gg 1$. Thus, figure 7(f) indicates that any deviation in the load resistance will cause a significant power drop in the SSHI case. Such an effect cannot be ignored in practical design, since there may exist other inherent electrical damping in the whole circuit system; for example, the diode loss is not taken into account in the present analysis. As a result, there seems to be no obvious advantage in using the SSHI electronic interface from the comparison between figures 7(c) and (f).

\section{Conclusions}

The electrical behavior of the piezoelectric power harvesting system using the SSHI electronic interface is analyzed and compared to that using the standard interface. Instead of using the un-coupled or in-phase assumptions to estimate the harvested power, an analytic expression of it based on the improved analysis is proposed explicitly and validated numerically for the SSHI circuit system. It is found that no matter whether the real electromechanical system is weakly or strongly coupled, the electrical response using an ideal SSHI interface is similar to that using the standard interface in a strongly coupled electromechanical system operated at the short circuit resonance. As a result, the harvested power increases tremendously for any weak coupling SSHI system at the cost of using a much larger optimal electric load.

The performance degradation due to the non-perfect voltage inversion is discussed and classified according to the relative strength of the coupling. This effect on power deduction is significant for weakly coupled electromechanical systems. On the other hand, if the electromechanical coupling is in the medium range, the degradation in harvested power is not pronounced in this case, and a new finding shows that the reduction in power is much less sensitive to frequency deviations than that using the standard technique. This provides a great advantage in design since the energy scavenger has a wider inherent bandwidth. Moreover, this frequency-insensitive feature is much more conspicuous when the inversion quality factor is improved. 

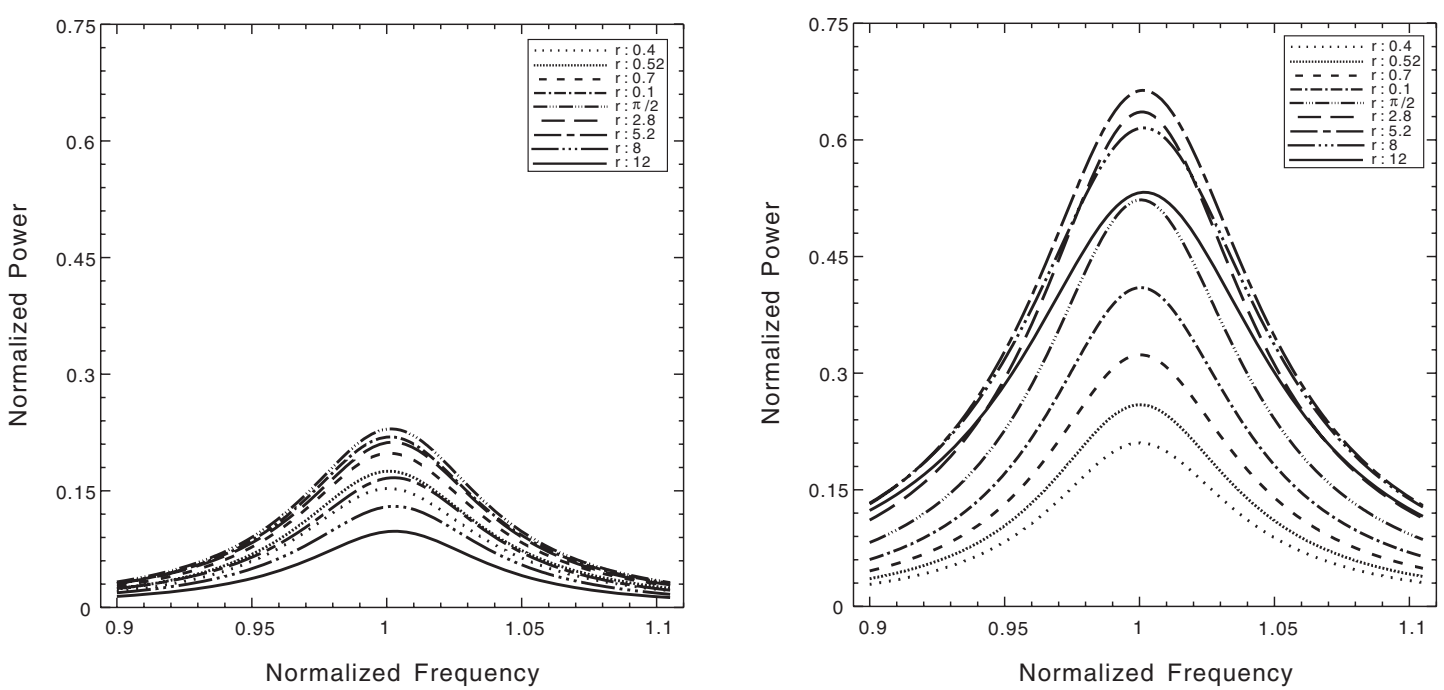

(a)

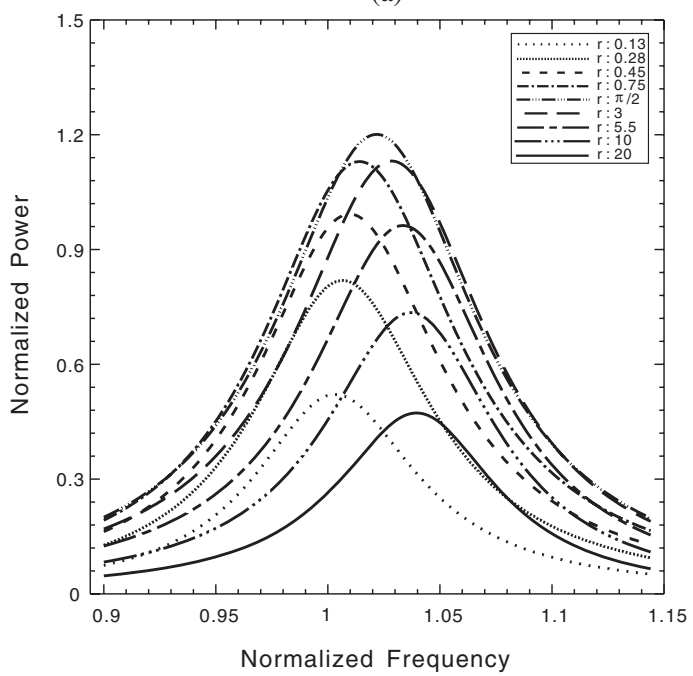

(d)

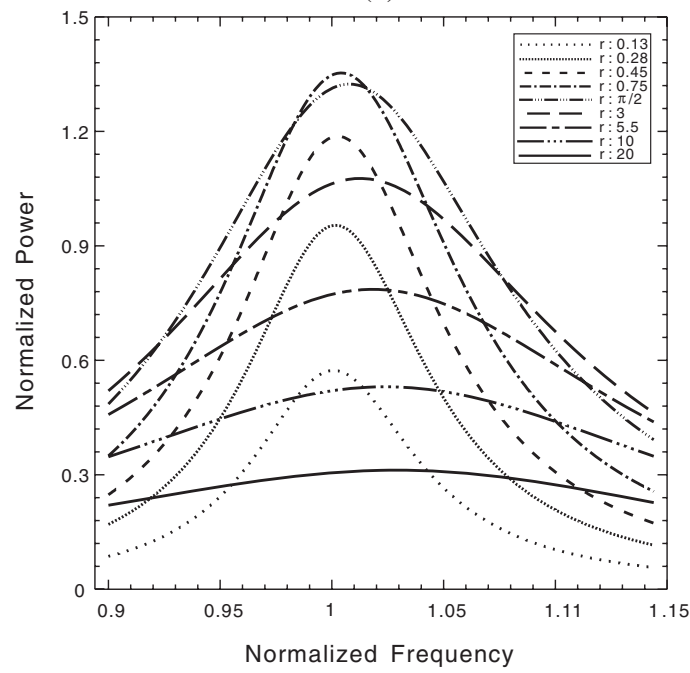

(b)

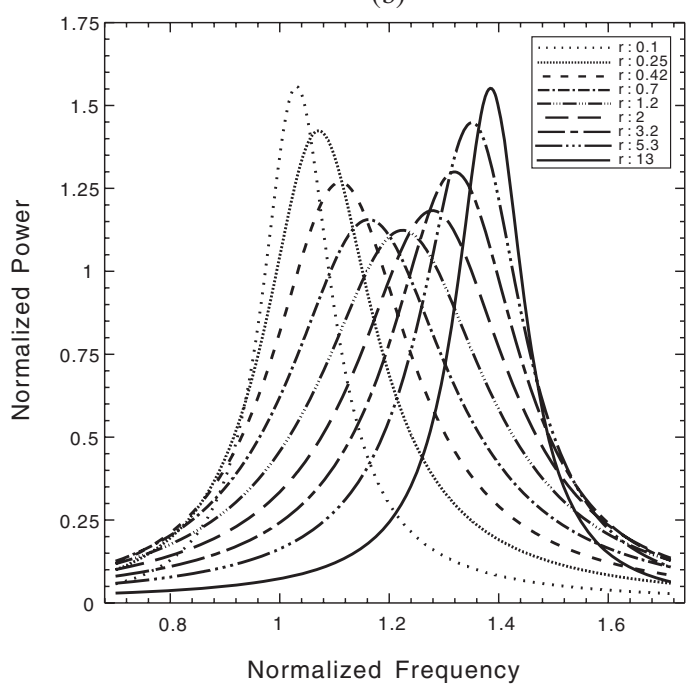

(c)

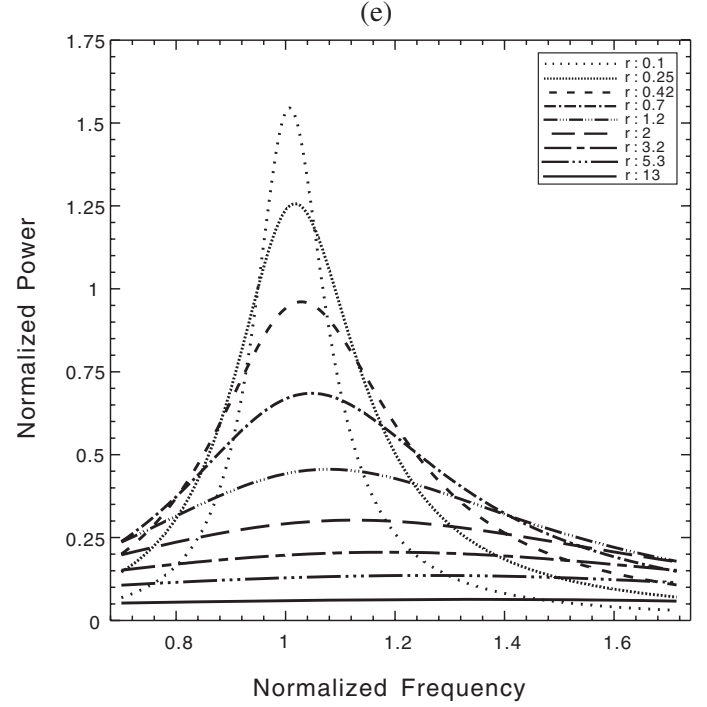

(f)

Figure 7. Normalized power versus frequency ratio for different values of normalized resistances. Notice that (a)-(c) are obtained using the standard electronic interface while (d)-(f) are obtained using the SSHI electronic interface. 


\section{Acknowledgments}

We thank Professors K C Wu, C K Lee and U Lei for their constant encouragement and support. We are glad to acknowledge partial support from the National Science Council under grant No 96-2628-E-002-119-MY3, and from the Ministry of Economic Affairs under grant No 95-EC-17A-05-S1-017 (WHAM-BioS).

\section{References}

[1] Ajitsaria1 J, Choe S Y, Shen D and Kim D J 2007 Modeling and analysis of a bimorph piezoelectric cantilever beam for voltage generation Smart Mater. Struct. 16 447-54

[2] Allen J J and Smits A J 2001 Energy harvesting EEL J. Fluids Struct. 15 629-40

[3] Badel A, Benayad A, Lefeuvre E, Lebrun L, Richard C and Guyomar D 2006 Single crystals and nonlinear process for outstanding vibration-powered electrical generators IEEE Trans. Ultrason. Ferroelectr. Freq. Control 53 673-84

[4] Badel A, Guyomar D, Lefeuvre E and Richard C 2005 Efficiency enhancement of a piezoelectric energy harvesting device in pulsed operation by synchronous charge inversion J. Intell. Mater. Syst. Struct. 16 889-901

[5] Badel A, Guyomar D, Lefeuvre E and Richard C 2006 Piezoelectric energy harvesting using a synchronized switch technique J. Intell. Mater. Syst. Struct. 17 831-9

[6] Charnegie D, Mo C, Frederick A A and Clark W W 2006 Tunable piezoelectric cantilever for energy harvesting Proc. 2006 ASME Int. Mechanical Engineering Congr. and Exposition IMECE2006-14431

[7] Cho J, Anderson M, Richards R, Bahr D and Richards C 2005 Optimization of electromechanical coupling for a thin-film PZT membrane: I. Modeling J. Micromech. Microeng. 15 1797-803

[8] Cho J, Anderson M, Richards R, Bahr D and Richards C 2005 Optimization of electromechanical coupling for a thin-film PZT membrane: II. Experiment J. Micromech. Microeng. 15 1804-9

[9] Cho J H, Richards R F, Bahr D F, Richards C D and Anderson M J 2006 Efficiency of energy conversion by piezoelectrics Appl. Phys. Lett. 89104107

[10] Choi W J, Jeon Y, Jeong J H, Sood R and Kim S G 2006 Energy harvesting MEMS device based on thin film piezoelectric cantilevers J. Electroceram. 17 543-8

[11] Cornwell P J, Goethal J, Kowko J and Damianakis M 2005 Enhancing power harvesting using a tuned auxiliary structure J. Intell. Mater. Syst. Struct. 16 825-34

[12] duToit N E and Wardle B L 2007 Experimental verification of models for microfabricated piezoelectric vibration energy harvesters AIAA J. $\mathbf{4 5} 1126-37$

[13] duToit N E, Wardle B L and Kim S G 2005 Design considerations for MEMS-scale piezoelectric mechanical vibration energy harvesters Integrated Ferroelectr. 71 121-60

[14] Elvin N G, Elvin A A and Spector M 2001 A self-powered mechanical strain energy sensor Smart Mater. Struct. $10293-9$

[15] Elvin N G, Lajnef N and Elvin A A 2006 Feasibility of structural monitoring with vibration powered sensors Smart Mater. Struct. 15 977-86

[16] Ericka M, Vasic D, Costa F, Poulin G and Tliba S 2005 Energy harvesting from vibration using a piezoelectric membrane J. Physique IV 128 187-93

[17] Fang H B, Liu J Q, Xu Z Y, Dong L, Chen D, Cai B C and Liu Y 2006 A MEMS-based piezoelectric power generator for low frequency vibration energy harvesting Chin. Phys. Lett. 23 732-4
[18] Flatau A B and Chong K P 2002 Dynamic smart material and structural systems Eng. Struct. 24 261-70

[19] Goldfarb M and Jones L D 1999 On the efficiency of electric power generation with piezoelectric ceramic Trans. ASME, J. Dynam. Syst. Meas. Control 121 566-71

[20] Guan M J and Liao W H 2007 On the efficiencies of piezoelectric energy harvesting circuits towards storage device voltages Smart Mater. Struct. 16 498-505

[21] Guyomar D, Badel A, Lefeuvre E and Richard C 2005 Toward energy harvesting using active materials and conversion improvement by nonlinear processing IEEE Trans. Ultrason. Ferroelectr. Freq. Control 52 584-95

[22] Horowitz S B, Sheplak M, Cattafesta L N III and Nishida T 2006 A MEMS acoustic energy harvester J. Micromech. Microeng. 16 S174-81

[23] Hu H P, Cao J G and Cui Z J 2007 Performance of a piezoelectric bimorph harvester with variable width J. Mech. 23 197-202

[24] Hu H P, Xue H and Hu Y T 2007 A spiral-shaped harvester with an improved harvesting element and an adaptive storage circuit IEEE Trans. Ultrason. Ferroelectr. Freq. Control 54 1177-87

[25] Jeon Y B, Sood R, Jeong J H and Kim S G 2005 MEMS power generator with transverse mode thin film PZT Sensors Actuators A 122 16-22

[26] Jiang S, Li X, Guo S, Hu Y, Yang J and Jiang Q 2005 Performance of a piezoelectric bimorph for scavenging vibration energy Smart Mater. Struct. 14 769-74

[27] Kim H W, Batra A, Priya S, Uchino K, Markley D, Newnham R E and Hofmann H F 2004 Energy harvesting using a piezoelectric 'cymbal' transducer in dynamic environment Japan. J. Appl. Phys. 43 6178-83

[28] Kim H W, Priya S, Uchino K and Newnham R E 2005 Piezoelectric energy harvesting under high pre-stressed cyclic vibrations J. Electroceram. 15 27-34

[29] Kim S, Clark W W and Wang Q M 2005 Piezoelectric energy harvesting with a clamped circular plate: analysis J. Intell. Mater. Syst. Struct. 16 847-54

[30] Kim S, Clark W W and Wang Q M 2005 Piezoelectric energy harvesting with a clamped circular plate: experimental study J. Intell. Mater. Syst. Struct. 16 855-63

[31] Lee C K, Hsu Y H, Hsiao W H and Wu J W J 2004 Electrical and mechanical field interactions of piezoelectric systems: foundation of smart structures-based piezoelectric sensors and actuators, and free-fall sensors Smart Mater. Struct. 13 1090-109

[32] Lefeuvre E, Badel A, Richard C and Guyomar D 2005 Piezoelectric energy harvesting device optimization by synchronous electric charge extraction J. Intell. Mater. Syst. Struct. 16 865-76

[33] Lefeuvre E, Badel A, Richard C, Petit L and Guyomar D 2006 A comparison between several vibration-powered piezoelectric generators for standalone systems Sensors Actuators A 126 405-16

[34] Leland E S and Wright P K 2006 Resonance tuning of piezoelectric vibration energy scavenging generators using compressive axial preload Smart Mater. Struct. 15 1413-20

[35] Lesieutre G A 1998 Vibration damping and control using shunted piezoelectric materials Shock Vib. Dig. 30 187-95

[36] Lesieutre G A, Ottman G K and Hofmann H F 2004 Damping as a result of piezoelectric energy harvesting J. Sound Vib. 269 991-1001

[37] Liao W H, Wang D H and Huang S L 2001 Wireless monitoring of cable tension of cable-stayed bridges using PVDF piezoelectric films J. Intell. Mater. Syst. Struct. 12 331-9

[38] Lu F, Lee H P and Lim S P 2004 Modeling and analysis of micro piezoelectric power generators for micro-electro-mechanical-systems applications Smart Mater. Struct. 13 57-63

[39] Makihara K, Onoda J and Miyakawa T 2006 Low energy dissipation electric circuit for energy harvesting Smart Mater. Struct. 15 1493-8 
[40] Mateu L and Moll F 2005 Optimum piezoelectric bending beam structures for energy harvesting using shoe inserts J. Intell. Mater. Syst. Struct. 16 835-45

[41] Ng T H and Liao W H 2005 Sensitivity analysis and energy harvesting for a self-powered piezoelectric sensor J. Intell. Mater. Syst. Struct. 16 785-97

[42] Ottman G K, Hofmann H F, Bhatt A C and Lesieutre G A 2002 Adaptive piezoelectric energy harvesting circuit for wireless remote power supply IEEE Trans. Power Electron. 17 669-76

[43] Ottman G K, Hofmann H F and Lesieutre G A 2003 Optimized piezoelectric energy harvesting circuit using step-down converter in discontinuous conduction mode IEEE Trans. Power Electron. 18 696-703

[44] Poulin G, Sarraute E and Costa F 2004 Generation of electric energy for portable devices: comparative study of an electromagnetic and a piezoelectric system Sensors Actuators A 116 461-71

[45] Priya S 2005 Modeling of electric energy harvesting using piezoelectric windmill Appl. Phys. Lett. 87184101

[46] Priya S, Chen C T, Fye D and Zahnd J 2005 Piezoelectric windmill: a novel solution to remote sensing Japan. J. Appl. Phys. 44 L104-7

[47] Richard C, Guyomar D, Audigier D and Ching G 1998 Semi passive damping using continuous switching of a piezoelectric device Proc. SPIE 3672 104-11

[48] Richards C D, Anderson M J, Bahr D F and Richards R F 2004 Efficiency of energy conversion for devices containing a piezoelectric component J. Micromech. Microeng. $14717-21$

[49] Roundy S 2005 On the effectiveness of vibration-based energy harvesting J. Intell. Mater. Syst. Struct. 16 809-23

[50] Roundy S, Leland E S, Baker J, Carleton E, Reilly E, Lai E, Otis B, Rabaey J M, Wright P K and Sundararajan V 2005 Improving power output for vibration-based energy scavengers IEEE Pervasive Comput. 4 28-36

[51] Roundy S and Wright P K 2004 A piezoelectric vibration based generator for wireless electronics Smart Mater. Struct. $131131-42$

[52] Roundy S, Wright P K and Rabaey J M 2004 Energy Scavenging for Wireless Sensor Networks with Special Focus on Vibrations (Boston, MA: Kluwer-Academic)
[53] Shahruz S M 2006 Design of mechanical band-pass filters with large frequency bands for energy scavenging Mechatronics 16 523-31

[54] Shenck N S and Paradiso J A 2001 Energy scavenging with shoe-mounted piezoelectrics IEEE Micro 21 30-42

[55] Shu Y C and Lien I C 2006 Analysis of power output for piezoelectric energy harvesting systems Smart Mater. Struct. 15 1499-512

[56] Shu Y C and Lien I C 2006 Efficiency of energy conversion for a piezoelectric power harvesting system $J$. Micromech. Microeng. 16 2429-38

[57] Shu Y C and Lien I C 2007 A comparison between the standard and SSHI interfaces used in piezoelectric power harvesting Proc. SPIE: Active and Passive Smart Struct. Integr. Syst. $\mathbf{6 5 2 5} 652509$

[58] Sodano H A, Inman D J and Park G 2005 Generation and storage of electricity from power harvesting devices J. Intell. Mater. Syst. Struct. 16 67-75

[59] Sodano H A, Lloyd J and Inman D J 2006 An experimental comparison between several active composite actuators for power generation Smart Mater. Struct. 15 1211-6

[60] Stephen N G 2006 On energy harvesting from ambient vibration J. Sound Vib. A 293 409-25

[61] Taylor G W, Burns J R, Kammann S M, Powers W B and Welsh T R 2001 The energy harvesting Eel: a small subsurface ocean/river power generator IEEE J. Oceanic Eng. 26 539-47

[62] Umeda M, Nakamura K and Ueha S 1996 Analysis of the transformation of mechanical impact energy to electric energy using piezoelectric vibrator Japan. J. Appl. Phys. 35 3267-73

[63] Umeda M, Nakamura K and Ueha S 1997 Energy storage characteristics of a piezo-generator using impact induced vibration Japan. J. Appl. Phys. 36 3146-51

[64] Wang Q M and Cross L E 1999 Constitutive equations of symmetrical triple layer piezoelectric benders IEEE Trans. Ultrason. Ferroelectr. Freq. Control 46 1343-51

[65] Williams C B and Yates R B 1996 Analysis of a micro-electric generator for microsystems Sensors Actuators A 52 8-11

[66] Yang J, Chen Z and Hu Y T 2007 An exact analysis of a rectangular plate piezoelectric generator IEEE Trans. Ultrason. Ferroelectr. Freq. Control 54 190-5 